\title{
Investigating the financial impact of coronavirus on leading stock indices
}

\author{
Mitashi Sinha', Ritvika Jalan', Rohini Singh² \\ ${ }^{1}$ Researcher, Shaheed Sukhdev College of Business Studies, New Delhi, India, ${ }^{2}$ Associate Professor, Shaheed Sukhdev \\ College of Business Studies, New Delhi, India.
}

\begin{abstract}
Purpose: The aim of this research paper is to investigate the short-term financial impact of coronavirus disease (COVID-19) on the leading stock markets indices of 20 countries, namely, India, the United States of America, Brazil, Russia, the United Kingdom, Spain, China, Germany, South Korea, France, Abu Dhabi, Taiwan, Thailand, Pakistan, New Zealand, Mexico, Japan, Canada, Singapore, and Australia. Methodology: A comparative and event study has been conducted between index closing prices of aforementioned countries during the COVID-19 outbreak (short-term and long-term basis) and pre-COVID period, keeping the S\&P Global 100 closing prices as the benchmark. For this, we have used statistical tools such as $t$-test and Mann-Whitney U-test and interpreted and analyzed the results thereof. Findings: Since the reaction of aforementioned stock indices has been analyzed under varying time periods for the event study, it was concluded that the announcement of the virus as a pandemic and its subsequent outbreak saw a sudden decrease in market returns, however, the prices recovered quickly. Implication: Even though COVID-19 initially affected investor confidence, it failed to create a long-term impact. Originality: Till now, no research has been conducted to test the effect of the WHO declaring COVID-19 a pandemic.
\end{abstract}

Key words: COVID-19, global equity markets, and event study, Mann-Whitney U-test, S\&P 100 index, $t$-test JEL Classification: G14 and F30

\section{INTRODUCTION}

On December 31, 2019, the first case of COVID-19 was in Wuhan, Hubei Province. Cases in China continued to rise despite a city-wide lockdown in Wuhan with around 2000 cases by the end of the month. The first case of COVID-19 outside China was recorded in Thailand on January 13, 2020. The number continued to rise exponentially with more than 100,000 cases by the $1^{\text {st }}$ week of March. On March 11, 2020, the WHO Director-General called a media briefing to state deep concern about both the alarming levels of spread and severity of the infection. Considering the on-going situation, the WHO made the assessment of declaring the novel coronavirus outbreak (2019-nCoV) a global pandemic and urged all countries to take urgent and aggressive action by building a comprehensive strategy to prevent infections, save lives, and minimize impact. (WHO, 2020).

The announcement attracted wide public and media attention which is expected to have affected investor sentiment. Fisher and Statman, 2000, stated "The relationship between the sentiment of individual investors and the sentiment of newsletter writers is strong." Major events such as natural disasters, terrorism threats, and political resolutions often have a great impact on investor sentiments which is

*Corresponding author: Rohini Singh, Associate Professor, Shaheed Sukhdev College of Business Studies,

New Delhi, India. E-mail: mitashi.19354@sscbs.du.ac.in

Received: 18 July 2020; Accepted: 28 August 2020

DOI: $10.18843 / \mathrm{ijcms} / \mathrm{v} 11 \mathrm{i} 3 / 02$

DOI URL: http://dx.doi.org/10.18843/ijcms/v11i3/02 
Sinha, et al.

reflected in the stock market returns. McGurk et al., (2020), found that "investor sentiment has a significant effect on abnormal stock returns."

COVID-19 is the most recently discovered coronavirus. Despite a low fatality rate, the number of deaths attributed to it far outweighs that from SARS or MERS due to the fact that it is significantly more infectious (Medical News Today, 2020).

Globalization has two intensifies the situation in two ways:

1. Globalization facilitates easy movement of goods and people, thereby increasing its frequency and volume. This also translates to easier spread of disease carrying microbes. Zappa et al. (2009) supported this argument in their paper.

2. It also increases the interdependency of global economies and world stock markets which poses a challenge for global investors to search for markets with low correlation and benefit from portfolio diversification. Siddiqui (2009) conducted a study using cointegration and Granger causality to prove high integration between the US and Asian markets with the exception of Japan where the integration varied between moderate to very high.

The unexpected situation created by COVID-19 gives us a unique opportunity to analyze the impact of a dreaded contagion and the fervor created by its global presence. In this paper, we examine the impact of COVID-19 on the stock market performance of 20 countries as represented by their respective leading stock indices.

\section{LITERATURE REVIEW}

The literature review of this project was conducted in the spring of March till June 2020. Due to COVID-19 being an ongoing pandemic, we have decided to keep the timeline of our paper from January 2019 to June 2020.

As per the Chief Economist of the International Monetary Fund (IMF), Gita Gopinath "The April edition of the World Economic Outlook projected a global growth in 2020 to fall to $-3 \%$. This is a downgrade of $6.3 \%$ points from January 2020, a major revision over a very short period" (IMF, 2020).

The Director of IMF and former CEO of World Bank, Kristalina Georgieva, declared in March 2020 that, "The outlook for global growth: For 2020 is negative - a recession at least as bad as during the global financial crisis or worse" (IMF, 2020).
Baker et al. (2020), theorized the comparison between the impact of the Spanish Flu and COVID-19 on the stock market. It states that "The current pandemic has grave implications for public health and for the economy. So, part of the answer surely lies in the severity of the pandemic, the apparent ease with which COVID-19 spreads, and the non-negligible mortality rate among those who contract the virus."

Barro et al., (2020), argued that extreme mitigation efforts such as widespread cancellations of travel and other major events - contribute further to the already depressed global economy.

Talking about the SARS pandemic, (Nippani and Washer, 2004), contradicted claims that it would have a tremendous negative impact on the affected counties. The paper concluded, "No evidence is found that SARS negatively impacted the main stock indices associated with Canada, Hong Kong, Indonesia, Philippines, Singapore, and Thailand."

Qiu et al. (2018) concluded that SARS eventually positively impacted China, by stating, "After SARS, China's public health emergency management system developed very fast, and as a consequence emergency management greatly improved."

It can be observed that the current pandemic has the most severe impact on the economy. Ozili and Thankom (2020) discussed the spillover effect of it on industries such as education, entertainment, health, travel, and sports. Liu et al. (2020) stated that "The outbreak has a significant negative effect on stock market returns across all affected countries and areas. Stock markets of Asian countries react more quickly to the outbreak with some of them recovering slightly in the later stage of the pandemic."

El-Erian (2020) states that the initial preventive measures which are being undertaken globally will have severe implications, "Measures such as "social distancing," separation, and isolation are inherently inconsistent with what drives economic growth, employment, and financial stability."

Talking about the sacred market forces, the pandemic has left both demand and supply in shambles. Elliot (2020) mentions, "The supply of goods and services is impaired because factories and offices are shut and output falls as a result. But demand also falls because consumers stay at home and stop spending, and businesses mothball investment." 
Sinha, et al.

\section{Statement of the Problem}

The black swan event has created much uncertainty and unrest among all stratas of the society. The global economy is in shambles, coupled with an increase in gloom ridden investor beliefs.

Keeping all these problems in mind, it was imperative that a comprehensive research is conducted to understand the actual impact of the pandemic, which is supported by statistical tools, accurate data, and correct facts.

\section{Objectives of the Study}

The objective of this paper is to,

1. Investigate the ramifications of COVID-19 on the stock indices of 20 countries,

2. Examine the difference in market returns of the representative composite indices,

3. Compare the event period returns for the 20 countries with the S\&P Global 100 Index using a one sample $t$-test,

4. Scan the median returns of the 20 indices using MannWhitney U-test on daily returns relative to S\&P 100,

5. Analyze the abnormal returns of 20 countries.

\section{Hypothesis}

Increased restriction on international trade and social distancing restrictions caused due to the pandemic are expected to affect investor sentiment which should be reflected in the representative indices of chosen countries.

Null hypothesis: The difference of location between the samples is equal to 0

Alternate hypothesis: The difference of location between the samples is different from 0 .

\section{Data Variables Used}

To examine the impact of COVID-19, the following indices were used: The CAC 40 Index to represent France, the SSE composite to represent the People's Republic of China, the IBEX 35 Index to represent Spain, the Global X DAX Germany ETF Index to represent Germany, the UK FTSE 100 Index to represent the United Kingdom, the NSEI Index to represent India, the S\&P 500 Index to represent the United States of America, the SGX (S68.SI) Index to represent Singapore, the BVSP Index to represent Brazil, the IMOEX. ME Index to represent Russia, the MXX Index to represent Mexico, the NZX 50 Index to represent New Zealand, the
S\&P/ASX 200 (AXJO) Index to represent Australia, the S\&P/TSX Composite Index to represent Canada, the KOSPI Index to represent South Korea, the ADX General Index to represent Abu Dhabi, the FTSE TWSE RAFI Taiwan 50 Index to represent Taiwan, the Nikkei 225 Index to represent Japan, the PAKS Index to represent Pakistan, and the SETI Index to represent Thailand.

The S\&P Global 100 market Index, an international index which measures the performance of blue chip companies present in global equity markets, is selected as the benchmark index for comparison and to calculate the abnormal returns of indices listed above.

The daily closing values of each of these indices were taken from reliable web portals such as Yahoo Finance and Investing.com.

Due to the WHO timeline and the aim of our paper, the entire study has been divided into four subperiods.

The first subperiod is defined from January 1, 2019, to December 30, 2019. This is called the "normal period" because in this time period, the virus was unknown and the cases were little to none. This period serves as a base for comparison and is a reflection of how the stock exchange would work in the pre-COVID times.

The second subperiod, defined from February 25, 2020, to March 11, 2020, is called the "pre-event window" because this signifies the period before WHO declared the Novel Coronavirus as a pandemic. The necessary assumption made here is that a negative impact can be seen on the stock indices of the affected countries and due to the news of the virus floating around.

The third subperiod, defined from March 12, 2020, to March 26, 2020, is called the "post-event window." It is a short-term period considered to evaluate the immediate impact of the WHOs declaration on stock indices worldwide.

The fourth subperiod is a long-term, post-event window. It is a 3-month long window, defined from March 12, 2020, to June 12,2020 . The assumption made for both the postevent windows is that the stock indices of affected countries will be severely negatively affected.

\section{METHODOLOGY}

After collection of data and their division into subperiods; the mean, standard deviation, and median returns were 
Sinha, et al.

calculated. Cross-sectional $t$-test and non-parametric Mann-Whitney U-test were performed to examine the daily returns of the stock indices and study the influence of the WHO announcement in different periods. For this, stock indices during the pre-event window, short event window, and long event window and then compared with the normal period. The above tests are also used to compare the performance of these indices during the four periods to the performance of the S\&P Global 100 Index. This is similar to the methodology used by Nippani and Washer, (2004).

In the second part of this paper, event study methodology is applied to examine the impact of the COVID-19 outbreak on stock returns. The methodology allows us to isolate the events from all others. This is done by predicting the expected stock returns in the absence of the event. Then, the difference between the expected and actual stock returns is used to calculate abnormal returns. Finally, cross-sectional t-test is used to analyze the impact of the event.

The parameter estimation period, prior to the date of the first recorded case of COVID-19, that is, December 31, 2019, extends for 200 days.

Data for daily returns are extracted for each observation in the sample. Expected returns for the stock indices are derived using the OLS (ordinary least squares) market model:

$$
R_{i, t}=\alpha_{i}+\beta_{i} R_{m t}+\varepsilon_{i, t}
$$

where,

$R_{i, t}=$ Return of index i

$R_{m t}^{i, t}=$ Market return on day $\mathrm{t}$ within the estimated window

$\alpha_{i}=$ Estimated intercept term for index i

$\beta_{i}=$ Estimated beta coefficient for index i

$\varepsilon_{i, t}=$ Statistic disturbance/Error term

Expected returns and abnormal are thereafter computed using the market model and OLS estimated parameters $\alpha_{i}$ and $\beta_{i}$ :

$$
\begin{aligned}
& E\left(R_{i, t}\right)=\alpha_{i}+\beta_{i} R_{m t} \\
& A R_{i, t}=R_{i, t}-E\left(R_{i, t}\right)
\end{aligned}
$$

where,

$E\left(R_{i, t}\right)=$ Expected return of index $\mathrm{i}$ on day $\mathrm{t}$

$R_{i, t}=$ Actual return of index $\mathrm{i}$ on day $\mathrm{t}$

$A R_{i, t}=$ Abnormal return of index $\mathrm{i}$ on day $\mathrm{t}$

The following formula is used to calculate the average abnormal return (AAR) of sample indices during time period t:(Fig.1)

$$
A A R_{t}=1 / N A R_{i, t}
$$

Where,

$\mathrm{t}=(0.1,2 \ldots)$

$\mathrm{N}=$ Total number of observations

Cumulative abnormal return (CAR) of index i during time period $t$ and cumulative average abnormal return (CAAR) is calculated as: (Fig.2)

$$
\begin{gathered}
C A R_{t}=A R_{i, t} \\
C A A R_{t}=A A R_{t}
\end{gathered}
$$

Cross-sectional $t$-test (CSect T) is given by:

$$
\text { tAARt }=\text { Rts giveSAARt }
$$

Where,

$\mathrm{m}=$ Mean of sample

$\mu=$ Mean of population

SAARt $=$ Standard Deviation of AAR during time period $t$

\section{FINDINGS AND DISCUSSION}

In the normal period, all indices showed positive mean returns, but only MXX, ADX General, and SETI display positive median returns. Returns of CAC 40, IBEX 35, UK FTSE 100, XJO, and IMOEX.ME significantly decreased during the pre-event window, while median returns of all composite indices except S68.SI are negative. Negative market returns were expected during the post-event window: Short term. However, the mean returns of CAC 40, UK FTSE 100, S68.SI, and IMOEX.ME were positive and the median returns of BVSP, IBEX 35, and S\&P 500 have increased by $2.77 \%, 2.55 \%$, and $2.36 \%$, respectively. In the long term, almost all representative composite indices, except SSE Composite, NSEI, S68.SI, and MXX, had positive mean returns. The U-values derived during this period clearly show that there were no significant negative deviations in the indices except S\&P TSX [Table 1a-d].

In the normal period, all indices showed positive mean and median returns except S\&P 100, MXX, ADX, and SETI., whereas in the pre-event window, in comparison to the global index, stock indices CAC 40, IBEX 35, IMOEX. ME, NIKKEI 225, BVSP, S\&P 500, XJO, S\&P TSX, and PAKS have performed below par. However, in both the post-event windows, long term and short term, a mixed reaction was witnessed and no significant changes can be seen [Table 2a-d]. 
Sinha, et al.

Table 1a: Mean and median returns during normal period

(January 1, 2019-December 30, 2019)

\begin{tabular}{lcccc}
\hline Index & Trading days & Mean (\%) & Standard deviation (\%) & Median (\%) \\
\hline CAC 40 & 253 & 0.10 & 0.84 & 0.17 \\
SSE Composite & 239 & 0.09 & 1.15 & 0.05 \\
IBEX 35 & 253 & 0.05 & 0.78 & 0.05 \\
UK FTSE 100 & 251 & 0.05 & 0.74 & 0.08 \\
DAX & 250 & 0.07 & 1.12 & 0.05 \\
NSEI & 239 & 0.06 & 0.88 & 0.04 \\
S\&P 500 & 250 & 0.10 & 0.79 & 0.10 \\
S68.SI & 243 & 0.09 & 1.04 & 0.11 \\
BVSP & 246 & 0.10 & 1.12 & 0.19 \\
IMOEX.ME & 249 & 0.10 & 0.71 & 0.09 \\
MXX & 243 & 0.02 & 0.84 & -0.04 \\
NZX 50 & 243 & 0.12 & 0.63 & 0.12 \\
XJO & 245 & 0.09 & 0.69 & 0.15 \\
S\&P TSX & 245 & 0.07 & 0.46 & 0.07 \\
KOSPI & 237 & 0.04 & 0.81 & 0.09 \\
ADX General & 191 & 0.03 & 0.91 & -0.03 \\
FTSE TWSE & 233 & 0.11 & 0.78 & 0.12 \\
Nikkei 225 & 232 & 0.09 & 0.88 & 0.10 \\
PAKS & 237 & 0.02 & 3.21 & 0.00 \\
SETI & 235 & 0.01 & 0.61 & -0.01 \\
\hline
\end{tabular}

Table 1b: Mean and median returns during pre-event window

(February 25, 2020-March 11, 2020)

\begin{tabular}{lcccccc}
\hline Index & Trading days & Mean (\%) & $\begin{array}{c}\text { Standard } \\
\text { deviation (\%) }\end{array}$ & Median (\%) & $\begin{array}{c}\text { t-value and } \\
\boldsymbol{P} \text {-value }\end{array}$ & $\begin{array}{c}\text { U-value and } \\
\boldsymbol{P} \text {-value }\end{array}$ \\
\hline CAC 40 & 12 & -1.85 & 2.73 & -1.71 & $-2.467(0.023)^{\star \star}$ & $825(0.008)$ \\
SSE composite & 12 & -0.16 & 2.00 & -0.24 & $-0.432(0.670)$ & $1324(0.654)$ \\
IBEX 35 & 12 & -1.97 & 2.62 & -2.50 & $-2.670(0.015)^{\star \star}$ & $872(0.013)$ \\
UK FTSE 100 & 12 & -1.60 & 2.64 & -1.51 & $-2.159(0.043)^{\star \star}$ & $994(0.047)$ \\
DAX & 12 & -1.52 & 3.21 & -1.19 & $-1.721(0.101)$ & $955(0.034)$ \\
NSEI & 11 & -1.10 & 1.87 & -0.46 & $-2.051(0.054)^{\star}$ & $780(0.023)$ \\
S\&P 500 & 12 & -1.27 & 4.02 & -2.26 & $-1.186(0.249)$ & $828(0.009)$ \\
S68.SI & 12 & -0.03 & 3.27 & 0.06 & $-0.127(0.899)$ & $1390(0.785)$ \\
BVSP & 10 & -2.68 & 6.00 & -2.58 & $-1.468(0.158)$ & $958(0.236)$ \\
IMOEX.ME & 11 & -1.94 & 2.90 & -0.69 & $-2.339(0.030)^{\star \star}$ & $740(0.01)$ \\
MXX & 12 & -1.00 & 2.53 & -1.24 & $-1.397(0.178)$ & $1013(0.074)$ \\
NZX 50 & 12 & -0.65 & 3.63 & -0.17 & $-0.727(0.475)$ & $1297(0.519)$ \\
XJO & 12 & -1.60 & 2.66 & -1.66 & $-2.198(0.040)^{\star *}$ & $742(0.004)$ \\
S\&P TSX & 12 & -1.66 & 3.50 & -1.62 & $-1.709(0.103)$ & $756(0.005)$ \\
KOSPI & 12 & -0.69 & 2.07 & -0.32 & $-1.228(0.234)$ & $1268(0.527)$ \\
\hline & & & & & & $($ Contd...)
\end{tabular}


Sinha, et al.

Table 1b: (Continued)

(February 25, 2020-March 11, 2020)

\begin{tabular}{lcccccc}
\hline Index & Trading days & Mean (\%) & $\begin{array}{c}\text { Standard } \\
\text { deviation (\%) }\end{array}$ & Median (\%) & $\begin{array}{c}\text { t-value and } \\
\boldsymbol{P} \text {-value }\end{array}$ & $\begin{array}{c}\text { U-value and } \\
\boldsymbol{P} \text {-value }\end{array}$ \\
\hline ADX & 10 & -1.40 & 4.69 & -0.60 & $-0.964(0.346)$ & $685(0.132)$ \\
FTSE TWSE & 11 & -0.50 & 1.32 & -1.00 & $-1.528(0.142)$ & $932(0.127)$ \\
Nikkei 225 & 12 & -1.52 & 2.01 & -1.68 & $-2.762(0.012)^{\star \star}$ & $755(0.008)$ \\
PAKS & 12 & -0.19 & 3.84 & -1.35 & $-0.186(0.853)$ & $1191(0.343)$ \\
SETI & 12 & -1.10 & 3.19 & -0.06 & $-1.201(0.244)$ & $1293(0.628)$ \\
\hline
\end{tabular}

*Significant at $5 \%$, ** significant at $2.5 \%$

\section{Table 1c: Mean and median returns during post-event window: Short term}

(March 12, 2020-March 26, 2020)

\begin{tabular}{lcccccc}
\hline Index & Trading days & Mean $(\%)$ & $\begin{array}{c}\text { Standard } \\
\text { deviation }(\%)\end{array}$ & Median (\%) & $\begin{array}{c}\boldsymbol{t} \text {-value and } \\
\boldsymbol{P} \text {-value }\end{array}$ & $\begin{array}{c}\text { U-value and } \\
\boldsymbol{P} \text {-value }\end{array}$ \\
\hline CAC 40 & 11 & 0.04 & 6.09 & 2.51 & $-0.032(0.974)$ & $1016(0.13)$ \\
SSE Composite & 11 & -0.63 & 1.96 & -0.98 & $-1.221(0.237)$ & $876(0.061)$ \\
IBEX 35 & 11 & -0.31 & 6.44 & 1.31 & $-0.184(0.855)$ & $1064(0.186)$ \\
UK FTSE 100 & 11 & 0.04 & 5.37 & 1.40 & $-0.007(0.994)$ & $1050(0.179)$ \\
DAX & 11 & -0.29 & 6.47 & 2.10 & $-0.183(0.856)$ & $899(0.052)$ \\
NSEI & 11 & -1.52 & 6.53 & -2.42 & $-0.800(0.433)$ & $1190(0.595)$ \\
S\&P 500 & 11 & -0.13 & 7.34 & 0.47 & $-0.104(0.917)$ & $1335(0.87)$ \\
S68.SI & 11 & 0.24 & 4.28 & 0.00 & $0.109(0.914)$ & $1261(0.753)$ \\
BVSP & 11 & -0.40 & 9.66 & 2.15 & $-0.171(0.865)$ & $1223(0.59)$ \\
IMOEX.ME & 11 & 0.10 & 4.87 & 1.31 & $-0.003(0.997)$ & $1260(0.654)$ \\
MXX & 10 & -0.74 & 3.55 & -1.79 & $-0.674(0.508)$ & $924(0.199)$ \\
NZX 50 & 11 & -0.23 & 7.74 & -0.67 & $-0.147(0.883)$ & $1084(0.289)$ \\
XJO & 11 & -0.87 & 5.76 & 0.70 & $-0.549(0.589)$ & $1260(0.716)$ \\
S\&P TSX & 11 & -0.30 & 7.89 & 1.77 & $-0.158(0.875)$ & $1225(0.61)$ \\
KOSPI & 11 & -0.97 & 5.66 & -3.19 & $-0.594(0.558)$ & $735(0.015)$ \\
ADX & 9 & -1.06 & 7.09 & -3.84 & $-0.460(0.650)$ & $759(0.554)$ \\
FTSE TWSE & 11 & -0.78 & 4.02 & -2.35 & $-0.729(0.474)$ & $878(0.078)$ \\
Nikkei 225 & 10 & -0.29 & 4.78 & -1.36 & $-0.250(0.804)$ & $826(0.123)$ \\
PAKS & 10 & -3.41 & 7.04 & -4.06 & $-1.541(0.139)$ & $783(0.07)$ \\
SETI & 11 & -1.07 & 5.75 & 0.92 & $-0.617(0.543)$ & $1143(0.517)$ \\
\hline
\end{tabular}

Table 1d: Mean and median returns during post-event window: Long term

(March 12, 2020-June 12, 2020)

\begin{tabular}{lcccccc}
\hline Index & Trading days & Mean (\%) & $\begin{array}{c}\text { Standard } \\
\text { deviation (\%) }\end{array}$ & Median (\%) & $\begin{array}{c}\boldsymbol{t} \text {-value and } \\
\boldsymbol{P} \text {-value }\end{array}$ & $\begin{array}{c}\text { U-value and } \\
\boldsymbol{P} \text {-value }\end{array}$ \\
\hline CAC 40 & 64 & 0.13 & 3.19 & 0.45 & $0.068(0.946)$ & $7209(0.176)$ \\
SSE composite & 63 & -0.02 & 1.10 & -0.02 & $-0.825(0.419)$ & $7086(0.473)$ \\
IBEX 35 & 64 & 0.02 & 3.25 & 0.35 & $-0.064(0.948)$ & $7462(0.333)$ \\
UK FTSE 100 & 63 & 0.10 & 2.87 & 0.55 & $0.142(0.888)$ & $6757(0.074)$ \\
DAX & 65 & 0.28 & 3.51 & 0.51 & $0.482(0.634)$ & $7252(0.182)$ \\
\hline
\end{tabular}


Sinha, et al.

Table 1d: (Continued)

(March 12, 2020-June 12, 2020)

\begin{tabular}{lcccccc}
\hline Index & Trading days & Mean (\%) & $\begin{array}{c}\text { Standard } \\
\text { deviation }(\%)\end{array}$ & $\begin{array}{c}\text { Median }(\%) \\
\boldsymbol{t} \text {-value and } \\
\boldsymbol{P} \text {-value }\end{array}$ & $\begin{array}{c}\text { U-value and } \\
\boldsymbol{P} \text {-value }\end{array}$ \\
\hline NSEI & 60 & -0.02 & 3.55 & 0.50 & $-0.158(0.875)$ & $6504(0.266)$ \\
S\&P 500 & 64 & 0.22 & 3.54 & 0.42 & $0.275(0.786)$ & $7199(0.216)$ \\
S68.SI & 62 & -0.07 & 2.82 & 0.15 & $-0.467(0.645)$ & $7401(0.831)$ \\
BVSP & 62 & 0.24 & 4.54 & 0.73 & $0.238(0.814)$ & $6960(0.288)$ \\
IMOEX.ME & 63 & 0.18 & 2.49 & 0.29 & $0.256(0.800)$ & $7232(0.339)$ \\
MXX & 61 & -0.02 & 2.28 & 0.12 & $-0.116(0.908)$ & $7270(0.818)$ \\
NZX 50 & 62 & 0.11 & 3.49 & 0.02 & $-0.010(0.991)$ & $7173(0.561)$ \\
XJO & 63 & 0.08 & 3.11 & 0.24 & $-0.008(0.993)$ & $7276(0.484)$ \\
S\&P TSX & 64 & 0.17 & 3.61 & 0.60 & $0.214(0.832)$ & $6498(0.035)$ \\
KOSPI & 58 & 0.23 & 2.81 & 0.25 & $0.513(0.613)$ & $6208(0.253)$ \\
ADX & 51 & 0.08 & 3.42 & 0.20 & $0.119(0.906)$ & $4436(0.328)$ \\
FTSE TWSE & 62 & 0.11 & 2.01 & 0.23 & $0.006(0.995)$ & $6725(0.404)$ \\
Nikkei 225 & 60 & 0.26 & 2.53 & 0.03 & $0.542(0.594)$ & $6805(0.79)$ \\
PAKS & 60 & 0.07 & 4.60 & 0.00 & $0.090(0.928)$ & $7088(0.971)$ \\
SETI & 60 & 0.21 & 2.91 & 0.62 & $0.546(0.590)$ & $5706(0.023)$ \\
\hline
\end{tabular}

Table 2a: Returns relative to global index during normal period

(January 1, 2019-December 30, 2019)

\begin{tabular}{|c|c|c|c|c|c|c|}
\hline Index & Trading days & Mean (\%) & $\begin{array}{c}\text { Standard } \\
\text { deviation (\%) }\end{array}$ & Median (\%) & $\begin{array}{l}t \text {-value and } \\
P \text {-value }\end{array}$ & $\begin{array}{c}U \text {-value and } \\
P \text {-value }\end{array}$ \\
\hline S\&P Global 100 & 253 & 0.01 & 1.72 & -0.09 & - & - \\
\hline CAC 40 & 253 & 0.10 & 0.84 & 0.17 & $2.012(0.058)^{\star}$ & $26,574(0.001)$ \\
\hline SSE Composite & 239 & 0.09 & 1.15 & 0.05 & $1.346(0.193)$ & $28,521(0.039)$ \\
\hline IBEX 35 & 253 & 0.05 & 0.78 & 0.05 & $1.125(0.274)$ & $28,214(0.021)$ \\
\hline UK FTSE 100 & 251 & 0.05 & 0.74 & 0.08 & $1.204(0.243)$ & $28,297(0.024)$ \\
\hline DAX & 250 & 0.07 & 1.12 & 0.05 & $1.088(0.289)$ & $27,704(0.009)$ \\
\hline NSEI & 239 & 0.06 & 0.88 & 0.04 & $1.108(0.281)$ & $29,220(0.102)$ \\
\hline S\&P 500 & 250 & 0.10 & 0.79 & 0.10 & $2.184(0.041)^{\star}$ & $26,487(0.001)$ \\
\hline S68.SI & 243 & 0.09 & 1.04 & 0.11 & $1.503(0.149)$ & $28,178(0.022)$ \\
\hline BVSP & 246 & 0.10 & 1.12 & 0.19 & $1.556(0.136)$ & $27,943(0.014)$ \\
\hline IMOEX.ME & 249 & 0.10 & 0.71 & 0.09 & $2.423(0.025)^{\star}$ & $27,578(0.007)$ \\
\hline MXX & 243 & 0.02 & 0.84 & -0.04 & $0.425(0.675)$ & $29,839(0.198)$ \\
\hline NZX 50 & 243 & 0.12 & 0.63 & 0.12 & $3.053(0.006)^{\star *}$ & $25,887(0)$ \\
\hline XJO & 245 & 0.09 & 0.69 & 0.15 & $2.075(0.051)$ & $26,472(0.001)$ \\
\hline S\&P TSX & 245 & 0.07 & 0.46 & 0.07 & $2.665(0.015)^{\star \star}$ & $26,699(0.001)$ \\
\hline KOSPI & 237 & 0.04 & 0.81 & 0.09 & $0.896(0.381)$ & $27,574(0.009)$ \\
\hline ADX & 191 & 0.03 & 0.91 & -0.03 & $0.512(0.614)$ & $26,875(0.044)$ \\
\hline FTSE TWSE & 233 & 0.11 & 0.78 & 0.12 & $2.193(0.040)^{\star}$ & $26,686(0.002)$ \\
\hline Nikkei 225 & 232 & 0.09 & 0.88 & 0.10 & $1.596(0.126)$ & $27,625(0.011)$ \\
\hline PAKS & 237 & 0.02 & 3.21 & 0.00 & $0.116(0.908)$ & $31,217(0.688)$ \\
\hline SETI & 235 & 0.01 & 0.61 & -0.01 & $0.293(0.772)$ & $28,826(0.066)$ \\
\hline
\end{tabular}

${ }^{*}$ Significant at $5 \%,{ }^{* *}$ significant at $1 \%$ 
Sinha, et al.

\begin{tabular}{|c|c|c|c|c|c|c|}
\hline \multicolumn{7}{|c|}{ (February 25, 2020-March 11, 2020) } \\
\hline Index & Trading days & Mean (\%) & $\begin{array}{c}\text { Standard } \\
\text { deviation (\%) }\end{array}$ & Median (\%) & $\begin{array}{c}t \text {-value and } \\
P \text {-value }\end{array}$ & $\begin{array}{l}U \text {-value and } \\
P \text {-value }\end{array}$ \\
\hline S\&P Global 100 & 12 & -0.10 & 0.11 & -0.17 & - & - \\
\hline CAC 40 & 12 & -1.85 & 2.73 & -1.71 & $-2.214(0.039)^{*}$ & $26,719(0.002)$ \\
\hline SSE Composite & 12 & -0.16 & 2.00 & -0.24 & $-0.096(0.924)$ & $28,629(0.063)$ \\
\hline IBEX 35 & 12 & -1.97 & 2.62 & -2.50 & $-2.473(0.022)^{\star}$ & $28,347(0.029)$ \\
\hline UK FTSE 100 & 12 & -1.60 & 2.64 & -1.51 & $-1.962(0.064)^{\star}$ & $28,155(0.022)$ \\
\hline DAX & 12 & -1.52 & 3.21 & -1.19 & $-1.536(0.140)$ & $27,477(0.007)$ \\
\hline NSEI & 11 & -1.10 & 1.87 & -0.46 & $-1.772(0.092)$ & $28,645(0.063)$ \\
\hline S\&P 500 & 12 & -1.27 & 4.02 & -2.26 & $-1.011(0.324)$ & $26,659(0.001)$ \\
\hline S68.SI & 12 & -0.03 & 3.27 & 0.06 & $0.0781(0.938)$ & $28,682(0.06)$ \\
\hline BVSP & 10 & -2.68 & 6.00 & -2.58 & $-1.360(0.189)$ & $28,444(0.037)$ \\
\hline IMOEX.ME & 11 & -1.94 & 2.90 & -0.69 & $-2.107(0.048)^{*}$ & $27,755(0.012)$ \\
\hline$M X X$ & 12 & -1.00 & 2.53 & -1.24 & $-1.237(0.230)$ & $29,666(0.201)$ \\
\hline NZX 50 & 12 & -0.65 & 3.63 & -0.17 & $-0.520(0.608)$ & $26,400(0.001)$ \\
\hline XJO & 12 & -1.60 & 2.66 & -1.66 & $-1.957(0.065)^{*}$ & $26,670(0.002)$ \\
\hline S\&P TSX & 12 & -1.66 & 3.50 & -1.62 & $-1.539(0.140)$ & $27,079(0.004)$ \\
\hline KOSPI & 12 & -0.69 & 2.07 & -0.32 & $-0.991(0.333)$ & $28,076(0.03)$ \\
\hline ADX & 10 & -1.40 & 4.69 & -0.60 & $-0.878(0.390)$ & $26,829(0.101)$ \\
\hline FTSE TWSE & 11 & -0.50 & 1.32 & -1.00 & $-1.013(0.323)$ & $26,545(0.002)$ \\
\hline Nikkei 225 & 12 & -1.52 & 2.01 & -1.68 & $-2.442(0.024)^{*}$ & $27,240(0.01)$ \\
\hline PAKS & 12 & -0.19 & 3.84 & -1.35 & $-0.080(0.936)$ & $31,298(0.847)$ \\
\hline SETI & 12 & -1.10 & 3.19 & -0.06 & $-1.086(0.290)$ & $29,385(0.184)$ \\
\hline
\end{tabular}

${ }^{*}$ Significant at $5 \%$

\section{Table 2c: Returns relative to global index during post-event window: Short term}

(March 12, 2020-March 26, 2020)

\begin{tabular}{lcccccc}
\hline Index & Trading days & Mean (\%) & $\begin{array}{c}\text { Standard } \\
\text { deviation (\%) }\end{array}$ & Median (\%) & $\begin{array}{c}\boldsymbol{t} \text {-value and } \\
\boldsymbol{P} \text {-value }\end{array}$ & $\begin{array}{c}\text { U-value and } \\
\boldsymbol{P} \text {-value }\end{array}$ \\
\hline S\&P Global 100 & 11 & -0.30 & 0.38 & -0.29 & - & - \\
CAC 40 & 11 & 0.04 & 6.09 & 2.51 & $0.183(0.856)$ & $27,393(0.006)$ \\
SSE Composite & 11 & -0.63 & 1.96 & -0.98 & $-0.561(0.581)$ & $28,777(0.08)$ \\
IBEX 35 & 11 & -0.31 & 6.44 & 1.31 & $-0.007(0.994)$ & $28,781(0.071)$ \\
UK FTSE 100 & 11 & 0.04 & 5.37 & 1.40 & $0.206(0.838)$ & $28,315(0.066)$ \\
DAX & 11 & -0.29 & 6.47 & 2.10 & $0.003(0.996)$ & $28,652(0.049)$ \\
NSEI & 11 & -1.52 & 6.53 & -2.42 & $-0.621(0.541)$ & $29,196(0.127)$ \\
S\&P 500 & 11 & -0.13 & 7.34 & 0.47 & $0.075(0.940)$ & $27,550(0.008)$ \\
S68.SI & 11 & 0.24 & 4.28 & 0.00 & $0.411(0.685)$ & $28,888(0.082)$ \\
BVSP & 11 & -0.40 & 9.66 & 2.15 & $-0.034(0.972)$ & $29,192(0.106)$ \\
IMOEX.ME & 11 & 0.10 & 4.87 & 1.31 & $0.267(0.792)$ & $28,600(0.045)$ \\
\hline
\end{tabular}

(Contd...) 
Sinha, et al.

\section{Table 2c: (Continued)}

(March 12, 2020-March 26, 2020)

\begin{tabular}{lcccccc}
\hline Index & Trading days & Mean (\%) & $\begin{array}{c}\text { Standard } \\
\text { deviation }(\%)\end{array}$ & Median (\%) & $\begin{array}{c}\boldsymbol{t} \text {-value and } \\
\boldsymbol{P} \text {-value }\end{array}$ & $\begin{array}{c}\text { U-value and } \\
\boldsymbol{P} \text {-value }\end{array}$ \\
\hline MXX & 10 & -0.74 & 3.55 & -1.79 & $-0.396(0.696)$ & $29,783(0.238)$ \\
NZX 50 & 11 & -0.23 & 7.74 & -0.67 & $0.028(0.977)$ & $26,522(0.001)$ \\
XJO & 11 & -0.87 & 5.76 & 0.70 & $-0.330(0.744)$ & $27,638(0.011)$ \\
S\&P TSX & 11 & -0.30 & 7.89 & 1.77 & $-0.003(0.996)$ & $28,073(0.024)$ \\
KOSPI & 11 & -0.97 & 5.66 & -3.19 & $-0.397(0.695)$ & $27,808(0.021)$ \\
ADX & 9 & -1.06 & 7.09 & -3.84 & $-0.323(0.749)$ & $27,223(0.179)$ \\
FTSE TWSE & 11 & -0.78 & 4.02 & -2.35 & $-0.399(0.694)$ & $26,704(0.003)$ \\
Nikkei 225 & 10 & -0.29 & 4.78 & -1.36 & $0.001(0.998)$ & $27,469(0.016)$ \\
PAKS & 10 & -3.41 & 7.04 & -4.06 & $-1.400(0.177)$ & $31,146(0.803)$ \\
SETI & 11 & -1.07 & 5.75 & 0.92 & $-0.444(0.661)$ & $29,842(0.303)$ \\
\hline
\end{tabular}

Table 2d: Returns relative to global index during post-event window: Long term

(March 12, 2020-June 12, 2020)

\begin{tabular}{lcccccc}
\hline Index & Trading days & Mean (\%) & $\begin{array}{c}\text { Standard } \\
\text { deviation }(\%)\end{array}$ & $\begin{array}{c}\text { Median }(\%) \\
\boldsymbol{t} \text {-value and } \\
\boldsymbol{P} \text {-value }\end{array}$ & $\begin{array}{c}\text { U-value and } \\
\boldsymbol{P} \text {-value }\end{array}$ \\
\hline S\&P Global 100 & 64 & 0.11 & 2.05 & -0.10 & - & - \\
CAC 40 & 64 & 0.13 & 3.19 & 0.45 & $0.049(0.961)$ & $28,344(0.311)$ \\
SSE Composite & 63 & -0.02 & 1.10 & -0.02 & $-0.918(0.369)$ & $27,361(0.284)$ \\
IBEX 35 & 64 & 0.02 & 3.25 & 0.35 & $-0.207(0.837)$ & $28,856(0.46)$ \\
UK FTSE 100 & 63 & 0.10 & 2.87 & 0.55 & $-0.015(0.987)$ & $29,044(0.723)$ \\
DAX & 65 & 0.28 & 3.51 & 0.51 & $0.399(0.693)$ & $28,342(0.394)$ \\
NSEI & 60 & -0.02 & 3.55 & 0.50 & $-0.268(0.791)$ & $28,682(0.71)$ \\
S\&P 500 & 64 & 0.22 & 3.54 & 0.42 & $0.265(0.793)$ & $27,916(0.245)$ \\
S68.SI & 62 & -0.07 & 2.82 & 0.15 & $-0.504(0.619)$ & $28,326(0.491)$ \\
BVSP & 62 & 0.24 & 4.54 & 0.73 & $0.233(0.817)$ & $27,994(0.303)$ \\
IMOEX.ME & 63 & 0.18 & 2.49 & 0.29 & $0.240(0.812)$ & $28,652(0.485)$ \\
MXX & 61 & -0.02 & 2.28 & 0.12 & $-0.426(0.674)$ & $28,768(0.65)$ \\
NZX 50 & 62 & 0.11 & 3.49 & 0.02 & $0.011(0.991)$ & $27,149(0.151)$ \\
XJO & 63 & 0.08 & 3.11 & 0.24 & $-0.065(0.948)$ & $27,723(0.265)$ \\
S\&P TSX & 64 & 0.17 & 3.61 & 0.60 & $0.137(0.892)$ & $28,327(0.491)$ \\
KOSPI & 58 & 0.23 & 2.81 & 0.25 & $0.333(0.742)$ & $27,695(0.318)$ \\
ADX & 51 & 0.08 & 3.42 & 0.20 & $-0.047(0.962)$ & $24,526(0.457)$ \\
FTSE TWSE & 62 & 0.11 & 2.01 & 0.23 & $-0.000(0.999)$ & $26,166(0.111)$ \\
Nikkei 225 & 60 & 0.26 & 2.53 & 0.03 & $0.475(0.639)$ & $26,398(0.135)$ \\
PAKS & 60 & 0.07 & 4.60 & 0.00 & $-0.059(0.953)$ & $28,396(0.646)$ \\
SETI & 60 & 0.21 & 2.91 & 0.62 & $0.275(0.785)$ & $28,325(0.684)$ \\
\hline
\end{tabular}

Mean abnormal returns and CAR of all composite indices in the normal period are positive. The median returns of all indices are also positive except SSE Composite, MXX, and
PAKS. Abnormal returns of CAC40, IBEX 35, UK FTSE 100, and DAX are substantially more than the global benchmark. The pre-event window showcases contrasting results. The 
Sinha, et al.

mean abnormal returns of all countries are negative, whereas median abnormal return of all composite indices except S68.SI and NZX 50 is positive. Analysis of the $t$-test and $P$-value shows that none of the changes in abnormal returns is significant in the short-term post-event window. The mean abnormal returns of all countries are positive except SSE Composite, DAX, S68.SI, MXX, and NZX 50 in the long-term post-event window. The median abnormal returns of three countries are negative - SSE Composite, NZX 50, and PAKS. The CAR of SSE Composite, UK FTSE 100,
DAX, S68.SI, MXX, and NZX 50 is negative. The t-test and p-value clearly show that there was no significant difference between the abnormal returns of the normal period and the long-term post-event period [Table 3a-d].

Table represents the average abnormal returns of the representative composite indices of the 20 selected countries from the day following the event $(\mathrm{T}+1)$ to the next 10 trading days. There was a significant decrease in returns on day $\mathrm{T}+1, \mathrm{~T}+3, \mathrm{~T}+5, \mathrm{~T}+8, \mathrm{~T}+9$, and $\mathrm{T}+10$ [Tables 4 and 5].

\begin{tabular}{lccccc}
\multicolumn{7}{c}{ Table 3a: Abnormal returns during normal period } \\
(January 1, 2019-December 30, 2019) \\
\hline Index & Trading days & Mean (\%) & Standard deviation (\%) & Median (\%) & CAR \\
\hline CAC 40 & 253 & 0.07 & 0.85 & 0.14 & 0.187 \\
SSE Composite & 239 & 0.04 & 1.14 & -0.01 & 0.090 \\
IBEX 35 & 253 & 0.09 & 0.83 & 0.15 & 0.219 \\
UK FTSE 100 & 251 & 0.09 & 0.84 & 0.16 & 0.227 \\
DAX & 250 & 0.09 & 0.84 & 0.17 & 0.227 \\
NSEI & 239 & 0.06 & 0.88 & 0.04 & 0.134 \\
S\&P 500 & 250 & 0.03 & 0.79 & 0.03 & 0.087 \\
S68.SI & 243 & 0.04 & 1.04 & 0.06 & 0.107 \\
BVSP & 246 & 0.06 & 1.12 & 0.15 & 0.155 \\
IMOEX.ME & 249 & 0.05 & 0.71 & 0.01 & 0.130 \\
MXX & 243 & 0.04 & 0.84 & -0.01 & 0.098 \\
NZX 50 & 243 & 0.03 & 0.63 & 0.03 & 0.078 \\
XJO & 245 & 0.06 & 0.69 & 0.13 & 0.149 \\
S\&P TSX & 245 & 0.04 & 0.46 & 0.05 & 0.097 \\
KOSPI & 237 & 0.03 & 0.81 & 0.06 & 0.064 \\
ADX & 191 & 0.05 & 0.91 & 0.00 & 0.105 \\
FTSE TWSE & 233 & 0.04 & 0.77 & 0.02 & 0.094 \\
Nikkei 225 & 232 & 0.04 & 0.87 & 0.04 & 0.103 \\
PAKS & 237 & 0.05 & 3.20 & -0.04 & 0.107 \\
SETI & 235 & 0.04 & 0.61 & 0.05 & 0.099 \\
\hline
\end{tabular}

\section{Table 3b: Difference in abnormal returns during pre-event window}

(February 25, 2020-March 11, 2020)

\begin{tabular}{lcccccc}
\hline Index & Trading days & Mean (\%) & Standard deviation (\%) & Median (\%) & CAR & $\boldsymbol{t}$-value and $\boldsymbol{P}$-value \\
\hline CAC 40 & 12 & -1.86 & 2.73 & -1.72 & -0.223 & $-2.453(0.032)^{\star \star}$ \\
SSE Composite & 12 & -0.25 & 2.03 & -0.26 & -0.030 & $-0.491(0.633)$ \\
IBEX 35 & 12 & -1.86 & 2.73 & -1.72 & -0.224 & $-2.470(0.031)^{\star \star}$ \\
UK FTSE 100 & 12 & -2.06 & 2.80 & -1.93 & -0.248 & $-2.664(0.022)^{\star \star}$ \\
DAX & 12 & -0.89 & 1.85 & -0.52 & -0.107 & $-1.837(0.093)^{\star}$ \\
NSEI & 11 & -1.10 & 1.87 & -0.45 & -0.121 & $-2.050(0.067)^{\star}$ \\
\hline
\end{tabular}

(Contd...) 
Sinha, et al.

\section{Table 3b: (Continued)}

(February 25, 2020-March 11, 2020)

\begin{tabular}{lcccccc}
\hline Index & Trading days & Mean (\%) & Standard deviation (\%) & Median (\%) & CAR & $t$-value and $P$-value \\
\hline S\&P 500 & 12 & -1.34 & 4.02 & -2.32 & -0.161 & $-1.185(0.260)$ \\
S68.SI & 12 & -0.08 & 3.27 & 0.01 & -0.009 & $-0.129(0.899)$ \\
BVSP & 10 & -2.72 & 5.99 & -2.62 & -0.272 & $-1.467(0.176)$ \\
IMOEX.ME & 11 & -1.99 & 2.89 & -0.74 & -0.219 & $-2.340(0.041)^{\star *}$ \\
MXX & 12 & -0.98 & 2.53 & -1.22 & -0.117 & $-1.393(0.190)$ \\
NZX 50 & 12 & -0.73 & 3.63 & -0.24 & -0.087 & $-0.723(0.484)$ \\
XJO & 12 & -1.63 & 2.66 & -1.68 & -0.195 & $-2.196(0.050)^{\star *}$ \\
S\&P TSX & 12 & -1.69 & 3.50 & -1.66 & -0.203 & $-1.708(0.115)$ \\
KOSPI & 12 & -0.70 & 2.07 & -0.33 & -0.085 & $-1.225(0.246)$ \\
ADX & 10 & -1.38 & 4.69 & -0.57 & -0.138 & $-0.965(0.359)$ \\
FTSE TWSE & 11 & -0.57 & 1.32 & -1.06 & -0.062 & $-1.521(0.159)$ \\
Nikkei 225 & 12 & -1.56 & 2.02 & -1.70 & -0.187 & $-2.737(0.019)^{\star *}$ \\
PAKS & 12 & -0.16 & 3.84 & -1.28 & -0.019 & $-0.181(0.859)$ \\
SETI & 12 & -1.06 & 3.19 & -0.04 & -0.127 & $-1.198(0.255)$ \\
\hline
\end{tabular}

${ }^{*}$ Significant at $5 \%,{ }^{* *}$ significant at $2.5 \%$

Table 3c: Difference in abnormal returns during post-event window: Short term

\begin{tabular}{lcccccc}
\multicolumn{7}{c}{ (March 12, 2020-March 26, 2020) } \\
\hline Index & Trading days $(\%)$ & Mean (\%) & $\begin{array}{c}\text { Standard } \\
\text { deviation }(\%)\end{array}$ & $\begin{array}{c}\text { Median } \\
(\%)\end{array}$ & CAR & t-value and $\boldsymbol{P}$-value \\
\hline CAC 40 & 11 & 0.02 & 6.09 & 2.53 & 0.002 & $-0.030(0.976)$ \\
SSE Composite & 11 & -0.67 & 1.97 & -1.03 & -0.074 & $-1.192(0.260)$ \\
IBEX 35 & 11 & 0.03 & 6.09 & 2.49 & 0.003 & $-0.032(0.755)$ \\
UK FTSE 100 & 11 & -0.80 & 5.83 & -0.59 & -0.088 & $-0.504(0.624)$ \\
DAX & 11 & -3.02 & 4.82 & -1.92 & -0.333 & $-2.145(0.057)$ \\
NSEI & 11 & -1.52 & 6.53 & -2.41 & -0.167 & $-0.798(0.443)$ \\
S\&P 500 & 11 & -0.19 & 7.34 & 0.42 & -0.021 & $-0.102(0.919)$ \\
S68.SI & 11 & 0.18 & 4.28 & -0.06 & 0.020 & $0.1051(0.918)$ \\
BVSP & 11 & -0.43 & 9.66 & 2.11 & -0.047 & $-0.169(0.868)$ \\
IMOEX.ME & 11 & 0.05 & 4.87 & 1.25 & 0.006 & $-7.335(0.999)$ \\
MXX & 10 & -0.71 & 3.54 & -1.74 & -0.071 & $-0.668(0.520)$ \\
NZX 50 & 11 & -0.30 & 7.73 & -0.69 & -0.033 & $-0.142(0.889)$ \\
XJO & 11 & -0.89 & 5.76 & 0.69 & -0.098 & $-0.548(0.595)$ \\
S\&P TSX & 11 & -0.33 & 7.89 & 1.73 & -0.037 & $-0.157(0.878)$ \\
KOSPI & 11 & -0.98 & 5.66 & -3.21 & -0.108 & $-0.591(0.566)$ \\
ADX & 9 & -1.03 & 7.09 & -3.81 & -0.093 & $-0.461(0.657)$ \\
FTSE TWSE & 11 & -0.83 & 4.02 & -2.40 & -0.092 & $-0.722(0.486)$ \\
Nikkei 225 & 10 & -0.31 & 4.78 & -1.34 & -0.031 & $-0.235(0.819)$ \\
PAKS & 10 & -3.36 & 7.05 & -4.00 & -0.336 & $-1.526(0.161)$ \\
SETI & 11 & -1.02 & 5.76 & 0.93 & -0.112 & $-0.610(0.554)$ \\
\hline
\end{tabular}


Sinha, et al.

\begin{tabular}{|c|c|c|c|c|c|c|}
\hline \multicolumn{7}{|c|}{ (March 12, 2020-June 12, 2020) } \\
\hline Index & Trading days & Mean (\%) & Standard deviation (\%) & Median & CAR & $t$-value and $P$-value \\
\hline CAC 40 & 64 & 0.09 & 3.18 & 0.004 & 0.058 & $0.043(0.965)$ \\
\hline SSE Composite & 63 & -0.09 & 1.07 & -0.001 & -0.055 & $-0.924(0.358)$ \\
\hline IBEX 35 & 64 & 0.09 & 3.19 & 0.003 & 0.058 & $0.009(0.992)$ \\
\hline UK FTSE 100 & 63 & 0.00 & 3.14 & 0.004 & -0.001 & $-0.234(0.815)$ \\
\hline DAX & 65 & -0.19 & 3.41 & 0.003 & -0.127 & $-0.675(0.501)$ \\
\hline NSEI & 60 & -0.02 & 3.54 & 0.005 & -0.013 & $-0.170(0.865)$ \\
\hline S\&P 500 & 64 & 0.15 & 3.54 & 0.004 & 0.099 & $0.270(0.788)$ \\
\hline S68.SI & 62 & -0.12 & 2.82 & 0.001 & -0.075 & $-0.458(0.647)$ \\
\hline BVSP & 62 & 0.20 & 4.54 & 0.007 & 0.123 & $0.233(0.815)$ \\
\hline IMOEX.ME & 63 & 0.13 & 2.48 & 0.001 & 0.082 & $0.247(0.804)$ \\
\hline$M X X$ & 61 & 0.00 & 2.27 & 0.002 & 0.001 & $-0.130(0.896)$ \\
\hline NZX 50 & 62 & 0.02 & 3.48 & 0.000 & 0.013 & $-0.026(0.978)$ \\
\hline XJO & 63 & -0.99 & 3.07 & -0.009 & -0.621 & $-2.701(0.008)$ \\
\hline S\&P TSX & 64 & 0.13 & 3.61 & 0.006 & 0.086 & $0.210(0.833)$ \\
\hline KOSPI & 58 & 0.21 & 2.80 & 0.002 & 0.123 & $0.504(0.615)$ \\
\hline ADX & 51 & 0.11 & 3.42 & 0.002 & 0.058 & $0.121(0.903)$ \\
\hline FTSE TWSE & 62 & 0.04 & 2.00 & 0.002 & 0.023 & $-0.014(0.988)$ \\
\hline Nikkei 225 & 60 & 0.21 & 2.52 & 0.000 & 0.126 & $0.508(0.613)$ \\
\hline PAKS & 60 & 0.09 & 4.62 & 0.000 & 0.051 & $0.067(0.946)$ \\
\hline SETI & 60 & 0.24 & 2.90 & 0.006 & 0.145 & $0.530(0.598)$ \\
\hline
\end{tabular}

Table 4: Abnormal returns on event day and 1 day after

\begin{tabular}{|c|c|c|c|c|}
\hline \multirow[t]{2}{*}{ Index } & \multicolumn{2}{|c|}{ Abnormal return } & \multirow[t]{2}{*}{$t$-value } & \multirow[t]{2}{*}{$P$-value } \\
\hline & Event day & 1 day after event & & \\
\hline CAC 40 & -0.005 & -0.123 & -1.107 & 0.468 \\
\hline SSE Composite & -0.010 & -0.015 & -4.837 & 0.130 \\
\hline IBEX 35 & -0.006 & -0.123 & -1.109 & 0.467 \\
\hline UK FTSE 100 & -0.084 & -0.015 & -1.459 & 0.382 \\
\hline DAX & 0.013 & -0.019 & -0.233 & 0.854 \\
\hline NSEI & 0.001 & -0.083 & -0.997 & 0.501 \\
\hline S\&P 500 & -0.050 & -0.096 & -3.197 & 0.193 \\
\hline S68.SI & -0.037 & -0.019 & -3.079 & 0.200 \\
\hline BVSP & -0.077 & -0.148 & -3.184 & 0.194 \\
\hline IMOEX.ME & -0.003 & -0.083 & -1.100 & 0.470 \\
\hline MXX & -0.055 & -0.099 & -3.555 & 0.175 \\
\hline NZX 50 & -0.022 & -0.053 & -2.554 & 0.238 \\
\hline XJO & -0.036 & -0.074 & -2.981 & 0.206 \\
\hline S\&P TSX & -0.046 & -0.124 & -2.219 & 0.270 \\
\hline KOSPI & -0.028 & -0.039 & -6.329 & 0.100 \\
\hline ADX & -0.006 & -0.074 & -1.189 & 0.445 \\
\hline FTSE TWSE & -0.011 & -0.039 & -1.838 & 0.317 \\
\hline Nikkei 225 & -0.024 & -0.044 & -3.342 & 0.185 \\
\hline PAKS & -0.019 & -0.065 & -1.856 & 0.315 \\
\hline SETI & -0.017 & -0.108 & -1.366 & 0.402 \\
\hline
\end{tabular}


Sinha, et al.

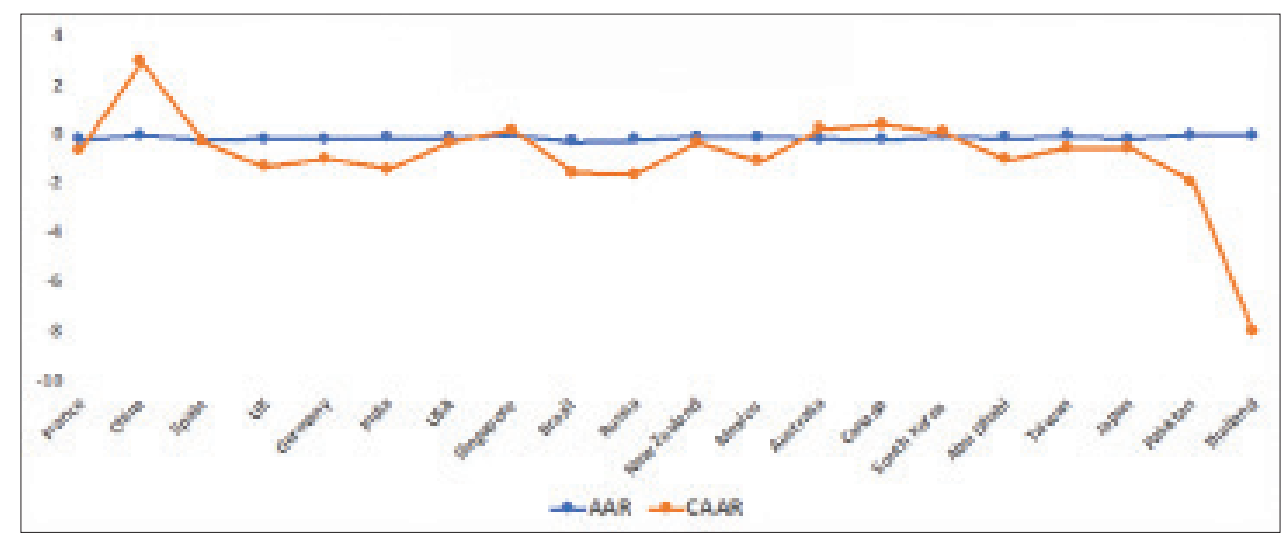

Figure 1: Daily pre-event plotting of AAR and CAAR for 20 countries (February 25, 2020-March 11, 2020)



Figure 2: Daily post-event plotting of AAR and CAAR for 20 countries (March 12, 2020-March 26, 2020)

\begin{tabular}{lccc}
\multicolumn{4}{c}{ Table 5: Abnormal return from $\mathrm{T}+\mathbf{1}$ to $\mathrm{T}+\mathbf{1 0}$} \\
Trading day & AAR & $\boldsymbol{t}$-value & $\boldsymbol{P}$-value \\
\hline $\mathrm{T}+1$ & 0.00015 & -7.865 & 0.0000 \\
$\mathrm{~T}+2$ & 0.00021 & 1.188 & 0.2490 \\
$\mathrm{~T}+3$ & 0.00027 & -8.996 & 0.0000 \\
$\mathrm{~T}+4$ & 0.00010 & 1.450 & 0.1630 \\
$\mathrm{~T}+5$ & 0.00017 & -4.025 & 0.0010 \\
$\mathrm{~T}+6$ & 0.00006 & -0.691 & 0.4980 \\
$\mathrm{~T}+7$ & 0.00013 & 2.037 & 0.0560 \\
$\mathrm{~T}+8$ & 0.00024 & -3.037 & 0.0070 \\
$\mathrm{~T}+9$ & 0.00021 & 4.071 & 0.0010 \\
$\mathrm{~T}+10$ & -0.00012 & 2.640 & 0.0160 \\
\hline
\end{tabular}

\section{CONCLUSION}

This research aimed at analyzing the effect of COVID-19 on the stock indices of 20 countries. An event study has also been conducted, taking WHOs declaration of the virus as a pandemic. This has been done panel wise, taking a pre-COVID, that is, normal period, a pre-event period, and two post-event periods. This research adds to the on-going literature as it discovers the highly anticipated outbreak effects on financial markets of this infectious disease.

Our findings suggest: (1) During the normal period, certain indices, namely, CAC 40, IBEX 35, UK FTSE 100 , and DAX have performed consistently well and even outperformed the global index, (2) A sharp decrease was seen in their performance due to the uncertainty hovering around the virus, increasing number of cases and the RussiaSaudi Arabia price war, (3) In most of the time periods, a general trend of underperformance by the indices of Asian countries has been seen, (4) A commonality among the 20 countries has been that each of the indices has witnessed volatility.

Despite controlling measures set in place by each country, the cases are still increasing at an exponential rate. 
The multiplier effects are also immeasurable, with laborintensive sectors being particularly hit.

A report suggested that as many as 1.6 billion of the world's 2 billion informal economy workers are affected by lockdown and containment measures (Business Standard, 2020).

However, economies around the world are now cautiously reopening, which is seemingly the need of the hour. Businesses must adapt to the "new normal." Business leaders should be agile and adept in leveraging on the first mover advantage.

Consumer facing businesses such as telecom, utilities, and retailers must practice strong customer relationship management. A possible roadmap for other businesses can be to focus on adapting innovative business models; innovation can be brought in any sphere, be it supply chain, delivery mechanisms, and many more.

Finally, we would like to state the limitations and further scope of study in our paper.

Since COVID-19 is an on-going pandemic, we had to restrict our date of study to June 12, 2020. Future developments and events might result in different findings and implications and will thus require distinct solutions. The demography of the investors was also not considered, such as age, gender, and income levels.

For further study, the date of the event can be changed to say January 20, 2020, when China confirmed humanto-human transmission of coronavirus (The Guardian, 2020).

\section{REFERENCES}

Baker, S.R., Bloom, N., Davis, S.J., Kost, K.J., Sammon, M.C., Viratyosin, T. (2020). The Unprecedented Stock Market Impact of COVID-19. Cambridge: The National Bureau of Economic Research.

Barro, R.J., Ursua, J.F., Weng, J. (2020). The Coronavirus and the Great Influenza Epidemic: Lessons from the "Spanish Flu" for the Coronavirus' Potential Effects on Mortality and Economic Activity. Washington, DC: The American Enterprise Institute.

Business Standard. (2020). Lockdown Worsening Poverty Levels among Workers in Informal Economy: ILO. Available from: https://www.business-standard. com/article/economy-policy/lockdown-worseningpoverty-levels-among-informal-economy-workersilo-120050900406_ 1.html. [Last accessed on $2020 \mathrm{Jul} 15]$.

El-Erian, M.A. (2020). The Coming Coronavirus Recession and the Uncharted Territory Beyond. Foreign Affairs. Available from: https://WWw.foreignaffairs.com/ articles/2020-03-17/coming-coronavirus-recession. [Last accessed on $2020 \mathrm{Jul} 17$ ].

Elliot, L. (2020). Prepare for the Coronavirus Global Recession. The Guardian. Available from: https:// www.theguardian.com/business/2020/mar/15/preparefor-the-coronavirus-global-recession. [Last accessed on $2020 \mathrm{Jul} 13$ ].

Fisher, K.L., Statman, M. (2000). Investor sentiment and stock returns, Financial Analysts Journal, 56(2), 16-23.

IMF. (2020). The Great Lockdown: Worst Economic Downturn since the Great Depression. Available from: https://Www.blogs.imf.org/2020/04/14/the-greatlockdown-worst-economic-downturn-since-the-greatdepression. [Last accessed on 2020 Jun 22].

IMF. (2020). Available from: https://www.imf.org/en/ News/Articles/2020/03/23/pr2098-imf-managingdirector-statement-following-a-g20-ministerial-callon-the-coronavirus-emergency; https://Www.imf.org/ external/index.htm. [Last accessed on 2020 Jun 22].

Liu, H.Y., Manzoor, A., Wang, C.Y., Zhang, L., Manzoor, Z. (2020). The COVID-19 outbreak and affected countries stock markets response, International Journal of Environmental Research and Public Health, 17(8), 2800.

McGurk, Z., Nowak, A., Hall, J.C. (2020). Stock returns and investor sentiment: Textual analysis and social media, Journal of Economics and Finance, 44, 458-485.

Medical News Today. (2020). Available from: https://www. medicalnewstoday.com/articles/how-do-sars-andmers-compare-with-covid-19\#Coronaviruses-pastand-present; https://Www.medicalnewstoday.com. [Last accessed on 2020 Jun 22].

Nippani, S., Washer, K.M. (2004). SARS: A non-event for affected countries' stock markets? Applied Financial Economics, 14(15), 1105-1110.

Ozili, P., Thankom, A. (2020). Spillover of COVID-19: Impact on the global economy, SSRN Electronic Journal, 1, 2139.

Qiu, W., Chu, C., Mao, A., Wu, J. (2018). The impacts on health, society, and economy of SARS and H7N9 outbreaks in China: A case comparison study, Journal of Environmental and Public Health, 2018, 2710185.

Siddiqui, S. (2009). Stock markets integration: Examining linkages between selected world markets. Vision, 13(1), 19-30.

The Guardian. (2020). China Confirms Human-to-human 
Sinha, et al.

Transmission of Coronavirus. Available from: https://WWw.theguardian.com/world/2020/jan/20/ coronavirus-spreads-to-beijing-as-china-confirmsnew-cases. [Last accessed on $2020 \mathrm{Jul} 18$ ].

WHO. (2020). Available from: https://Www.who. int $/ d g /$ speeches/detail/who-director-general-s -opening-remarks-at-the-media-briefing-on-covid-19 ---11-march-2020; https://www.who.int. [Last accessed on 2020 Jun 20].

Zappa, A., Amendola, A., Romanò, L., Zanetti, A. (2009). Emerging and re-emerging viruses in the era of globalisation. Blood Transfusion, 7(3), 167-171. 